\title{
Shape Analysis of the LHC Pre-Series Dipoles
}

\author{
Giuseppe Gubello, Marco La China, and Walter Scandale
}

\begin{abstract}
This paper presents the results of a comprehensive analysis, from the geometric point of view, of the pre-series LHC dipoles. The progressive change of the imposed magnet shape has been monitored from the first assembly stage until after the cold test. Data concerning the error on sagitta, extremity positions and sextupolar corrector positions are provided for the pre-series magnets. Implications of aligning out-of-tolerance dipoles by the extremities are also discussed.
\end{abstract}

Index Terms-Corrector positions, dipole alignment, dipole shape.

\section{INTRODUCTION}

$\mathbf{T}$ HE Large Hadron Collider is currently under construction at CERN. Two counter rotating beams will provide proton-proton collisions at $7 \mathrm{TeV}$ center mass energy. One of the major efforts toward the accelerator completion is the manufacturing of the 1232 superconducting magnets required to bend the particle beams along the $27 \mathrm{~km}$-long circular trajectory. The manufacturing procedure of each $15 \mathrm{~m}$-long, $0.5 \mathrm{~m}$-wide and 27 tons-mass magnet is strongly driven by tight geometrical tolerances due to beam dynamics. In order to ensure the maximum transverse clearance for circulating particles with the minimum coil size, the central part of the magnet is bent in the horizontal plane by about $5 \mathrm{mrad}$. In addition, to compensate systematic high order field harmonics, multipolar correctors are placed at one or both ends of each dipole. In order to satisfy the geometrical tolerances imposed on the dipole shape, the axis of the central part should be enclosed in a torus of $1 \mathrm{~mm}$ minor radius and the ends in two cylinders of $0.6 \mathrm{~mm}$ radius respect to the geometric axis of the dipole. On the other hand the correctors should stay in average within $0.3 \mathrm{~mm}$ from the geometric axis with an $\mathrm{rms}$ error not in excess of $0.5 \mathrm{~mm}$, see [1].

The most critical process is the bending of the cylindrical structure of the magnet in the horizontal plane (see also [2]). The curved shape imposed by the welding press, must indeed be preserved throughout the rest of the assembly, transport, testing stages (from now called pre-operative stages), the positioning in the tunnel and the start of the operative stages.

The change of the imposed curvature, experienced by some of the magnets manufactured up to now, leads to launch a comprehensive analysis of the magnet shape throughout the successive pre-operative stages. Magnets undergo a bending procedure that is associated with the welding of the shrinking cylinder.

This paper presents also the geometric measurements performed on pre-series dipoles. The statistical analysis is described and the results are discussed.

Manuscript received October 20, 2003.

The authors are with CERN (European Center for Nuclear Research) $\mathrm{CH}$ 1211 Geneve, Switzerland (e-mail: giuseppe.gubello@cern.ch).

Digital Object Identifier 10.1109/TASC.2004.829055

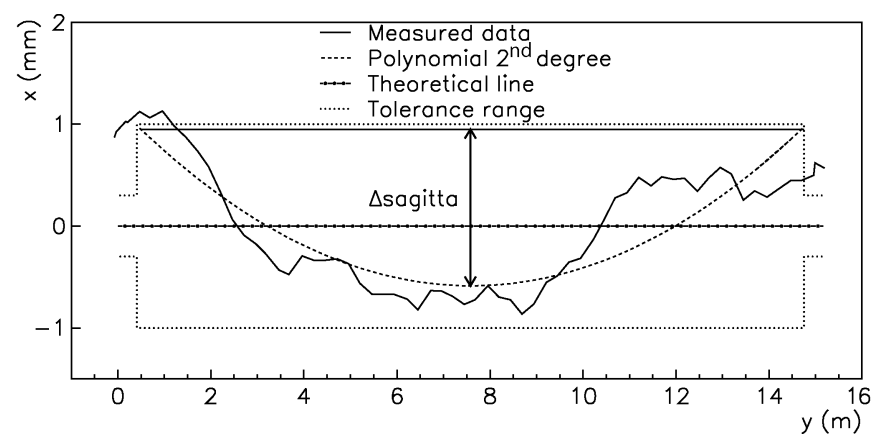

Fig. 1. Sagitta error estimation of a typical measurement.

\section{Geometric Shape, Referential AND MeAsurements}

In a dipole the geometrical axes (also referred to as theoretical or ideal) are defined to be the two counter-rotating beams at nominal LHC energies. At room temperature the inter axes distance is $194.35 \mathrm{~mm}$ and at the operating temperature $(1.9 \mathrm{~K})$ it shrinks to $194 \mathrm{~mm}$. The mechanical axes are identified by the cold bore tube centers whereas the magnetic ones by the superconducting coil centers. The central part, $14343 \mathrm{~mm}$ long, is bent by about $5 \mathrm{mrad}$ with a theoretical radius of $2812360 \mathrm{~mm}$, and it is prolonged beyond the extremities by two straight parts of $408.5 \mathrm{~mm}$. To describe the dipole shape in 3D space a reference system is defined as follow: for each aperture the $\mathrm{Y}$ axis is the one joining the two extremities of the geometric axis, the connection extremity being the origin. The $\mathrm{X}$ axis is the one passing at the origin, orthogonal to the $\mathrm{Y}$ axis and the positive direction points toward the dipole center of curvature. Finally the $\mathrm{Z}$ axis is orthogonal to $\mathrm{X}$ and $\mathrm{Y}$, passes through the origin and the positive direction is opposite to that of gravity.

Dipole axis measurements, carried out using a laser tracker and a reflecting mole pulled along the apertures [3], are given in terms of displacements from the theoretical shape. A typical measurement of one aperture profile in the horizontal plane, is shown in Fig. 1. The abscissa axis is the curvilinear coordinate of the theoretical axis and the solid curve represents the measured errors of the mechanical axis with respect to the ideal one. The distance is always computed in the $(\mathrm{X}, \mathrm{Z})$ plane orthogonal to the $\mathrm{Y}$ axis. To minimize the noise associated to the measurement itself and to integrate in an analytic formula the large amount of the treated data a polynomial fit is used. To preserve a high accuracy level, the polynomial is of 10th degree.

To monitor the evolution of the shape error across the magnet pre-operative stages, geometric measurements are performed at the following steps:

- After Welding: cold mass shape just after bending and welding of shrinking cylinder;

- After Manufacturing: cold mass shape at the last stage in the factory (i.e., end cover welded); 

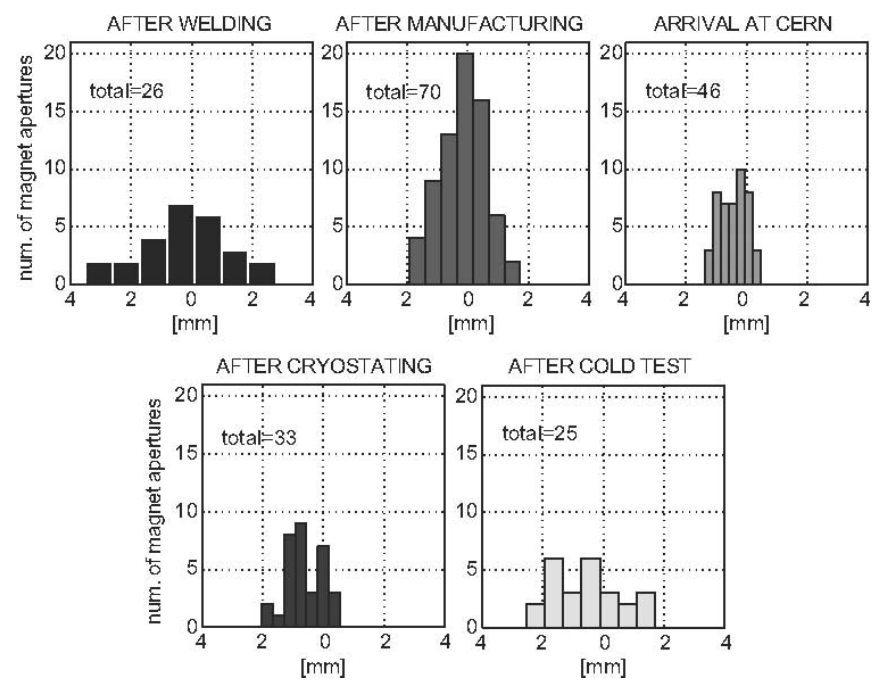

Fig. 2. Distribution of sagitta errors for inner tube.

- Arrival at CERN: after the transport from the factory;

- After Cryostating: first measurement of the cold mass inside its cryostat;

- After Cold Test: after power tests and the thermal cycles (300 K-1.9 K-300 K).

To simplify our analysis we will consider only two parameters to characterize the dipole geometrical properties, i.e., the errors on sagitta and the position of the dipole ends (and that of the sextupolar correctors) in the vertical and in the horizontal plane.

The results of a statistical analysis on these parameters, performed on 70 dipoles produced up to now, are given in the following sections.

\section{ERRORS ON SAGITTA}

A crucial parameter in the geometrical analysis of the dipole is the sagitta of the bent part, whose nominal value at room temperature is $9.14 \mathrm{~mm}$. Indeed in a dipole with a nonnominal sagitta the available mechanical clearance for the particle beams is reduced.

To compute the error on the sagitta, the shape errors measured in the bent part of the dipole are approximated by a 2nd order polynomial fit as shown in Fig. 1. The sagitta error is the sagitta of the interpolating curve in the domain of interest, i.e., along the curved part of the dipole. With our conventions, the sagitta error ( $\Delta$ sagitta) in Fig. 1 is negative and corresponds to a dipole more curved than the nominal one.

The histograms in Fig. 2 give, the distribution of the sagitta error in the consecutive stages from just 'after welding' up to 'after cold test'. The results for the inner and the outer tube are statistically very close. It can be noticed that the wide spread of sagitta errors in the 'after welding' stage are squeezed considerably in the 'after manufacturing' stage. At this stage the dipoles are ready for shipping to CERN and have undergone, for these concerned, the reshaping operation. On the contrary, in 'after cold test' stage the distribution spread is again larger and similar to the 'after welding' stage. The corresponding numerical values for averages and standard deviations are reported in Table I. It can be noticed that the average value of the error is slightly negative in 'after welding' and 'after manufacturing'
TABLE I

AVERAGE AND STANDARD DEVIATION OF SAGITTA ERRORS

\begin{tabular}{lccc}
\hline \hline & Dipoles & Average [mm] & St. dev. [mm] \\
\hline After welding & 26 & -0.14 & 1.48 \\
After manufacturing & 70 & -0.16 & 0.75 \\
Arrival at CERN & 46 & -0.43 & 0.45 \\
After cryostating & 33 & -0.62 & 0.64 \\
After cold test & 24 & -0.57 & 1.11 \\
\hline \hline
\end{tabular}

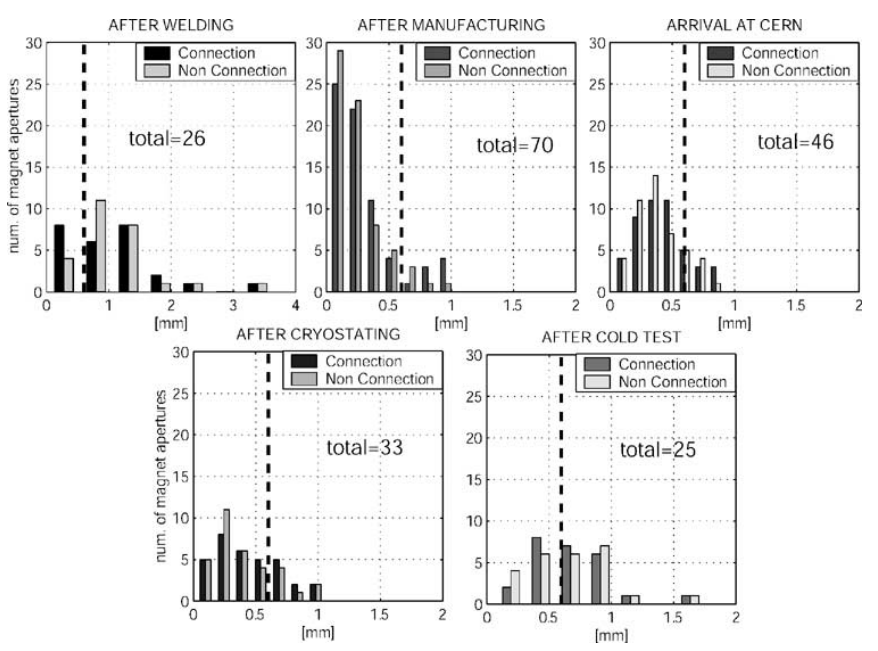

Fig. 3. Distribution all over the magnet production of tube end displacements in radial direction. Note the different $\mathrm{X}$-axis scale between graphs.

stage and decreases rather considerably afterward. This is a statistical feature related to the unequal sample considered at the various stages. By selecting the subsample for which all stages of measurement are available we see that the dipoles in average are too curved 'after welding' well corrected 'after manufacturing' and tend to recover the 'after welding' curvature in the successive operations.

\section{END DisPlacements AND CORRECTOR POSITION}

The cold bore tube end displacements for both inner and outer tube are defined as the displacements at $\mathrm{y}=0$ and $\mathrm{y}=15158 \mathrm{~mm}$, obtained from the 10th order interpolating shape. The histogram in Fig. 3 represents the radial distribution of the tube end displacements from the theoretical position as measured during the manufacturing phases. The dotted vertical line, at $0.6 \mathrm{~mm}$ is the tolerance limit. Averages and $\mathrm{rms}$ errors are given in Table II.

One can notice that the average value is large in 'after welding' it is condensed in 'after manufacturing' due to the centering of the cold bore tube by welding the end cover; then it progressively increases up 'after cold test' where the average value is sligthly out of tolerance.

In each produced dipole, to compensate the effect of sextupolar distortions on beam stability induced by persistent currents, yoke saturation and coil deformation, a sextupolar corrector magnet (MCS) is embedded at the nonconnection end. Since in the available sample of dipoles, no decapolar/octupolar correctors are present yet except few exceptions, we will concentrate our attention on the sextupole corrector position which 
TABLE II

Average and Standard Deviation of Radial DISPLACEMENTS FOR END POSITION

\begin{tabular}{|c|c|c|c|c|}
\hline & & Dipoles & "Average [mm] & $\overline{\text { St. dev. }[\mathrm{mm}]}$ \\
\hline \multirow{5}{*}{ 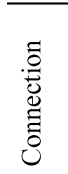 } & After Welding & 26 & 1.06 & 0.72 \\
\hline & After Manufacturing & 70 & 0.28 & 0.23 \\
\hline & Arrival at CERN & 46 & 0.40 & 0.19 \\
\hline & After Cryostating & 33 & 0.44 & 0.26 \\
\hline & After Cold Test & 24 & 0.66 & 0.31 \\
\hline \multirow{5}{*}{ ż } & After Welding & 26 & 1.09 & 0.68 \\
\hline & After Manufacturing & 70 & 0.32 & 0.20 \\
\hline & Arrival at CERN & 46 & 0.40 & 0.19 \\
\hline & After Cryostating & 33 & 0.45 & 0.23 \\
\hline & After Cold Test & 24 & 0.68 & 0.33 \\
\hline
\end{tabular}

TABLE III

AVERAGE AND STANDARD DEVIATION FOR COMPUTED SEXTUPOLE POSITION FOR BOTH APERTURES

\begin{tabular}{|c|c|c|c|c|}
\hline & & Dipoles & Average [mm] & St. dev. $[\mathrm{mm}]$ \\
\hline \multirow{4}{*}{ 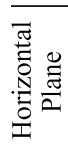 } & After Manufacturing* & 69 & 0.01 & 0.09 \\
\hline & Arrival at CERN & 42 & 0.21 & 0.26 \\
\hline & After Cryostating & 30 & 0.20 & 0.30 \\
\hline & After Cold Test & 23 & 0.17 & 0.62 \\
\hline \multirow{4}{*}{ 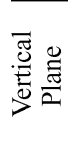 } & After Manufacturing* & 69 & 0.02 & 0.06 \\
\hline & Arrival at CERN & 42 & -0.08 & 0.29 \\
\hline & After Cryostating & 30 & 0.05 & 0.19 \\
\hline & After Cold Test & 23 & 0.07 & 0.20 \\
\hline
\end{tabular}

${ }^{*}$ Directly measured data.

is a crucial parameter affecting the machine overall performance and must be monitored during all the pre-operative phases. Actually, after the complete manufacturing of the dipole, the sextupole corrector is no longer accessible for mechanical measurements so that its position can only be inferred through the mapping of its magnetic field. Since, for practical reasons, such a method is not available at all measuring stages, a more convenient method is used based on available mechanical measurements. The main assumption on which it is based is the absence of any relative motion between the sextupole and the relative cold bore aperture. Thus, operatively, it is necessary to measure the distance between cold bore axis and sextupole axis only once in order to be able to get the sextupole position from any successive cold bore axis measurement. This distance is directly measured for all the dipoles in the 'after manufacturing' stage.

In all the following stages the sextupole corrector position is deduced by appropriate interpolation of the dipole shape at the longitudinal position of the corrector, i. e., of $15033 \mathrm{~mm}$ along the aperture axis. The sextupole positions computed for each different pre-operative stage are shown in Table III.

The results show that the average values are in tolerance for all pre-operative stages; regarding the standard deviation only 'after cold test' value is exceeding the tolerance of $0.5 \mathrm{~mm}$. The analysis results are given in the polar diagrams of Fig. 4 in which the $0.3 \mathrm{~mm}$ radius circle represents the imposed tolerance while the $1.5 \mathrm{~mm}$ radius one indicates three times the tolerance of the standard deviation.

In some cases the dipole ends and the spool pieces are out of tolerance. To recover these dipoles without repairs we plan to align them by the extremities. A possible strategy for this
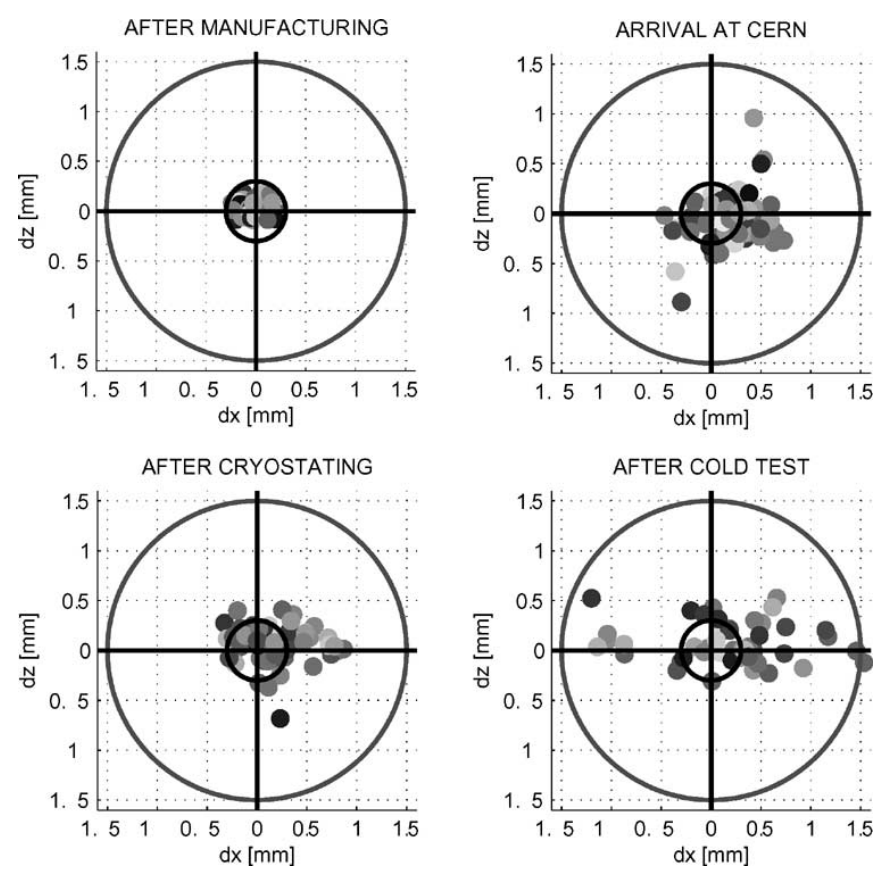

Fig. 4. Position of sextupolar corrector centers as computed in the pre-operative stages.

TABLE IV

AVERAGE AND STANDARd DEVIATION OF RADIAL DisPlacemENTS OF END POSITION FOR DiPOLES WITH NeW ALIGNMENT

\begin{tabular}{lccccc}
\hline \hline & & \multicolumn{2}{c}{ Connection } & \multicolumn{2}{c}{ Non Connection } \\
& Dipoles & Av. & St.Dev. & Av. & St.Dev. \\
\hline After welding & 26 & 0.66 & 0.56 & 0.70 & 0.62 \\
After manufacturing & 70 & 0.34 & 0.21 & 0.39 & 0.25 \\
Arrival at CERN & 46 & 0.25 & 0.14 & 0.30 & 0.15 \\
After cryostating & 33 & 0.27 & 0.18 & 0.32 & 0.17 \\
After cold test & 25 & 0.33 & 0.19 & 0.35 & 0.17 \\
\hline \hline
\end{tabular}

operation is outlined in Section IV-A, where the consequences for the already built dipoles are statistically investigated.

\section{A. Novel Alignment}

Since in each magnet, for geometrical reasons, the extremities and the central part show horizontal misalignments that are opposite with respect to the theoretical curve, the question is to find an optimum balance. One possibility is to align the magnet on the ideal trajectory by the extremities so that the interconnections are closer to the ideal position whereas the center moves away.

On the other hand, the non linear feed-down effect of systematic harmonics is compensated at the first order whenever nonlinear correctors are installed along the beam orbit, with the currently used assembly procedure. By displacing the horizontal alignment with a radial shift of about one half of the sagitta error, the already built dipoles will have extremities distributed around the nominal position as shown in Table IV and correctors as in Table V.

The maximum displacement in the dipole central part which increases from $1.8 \mathrm{~mm}$ (with standard alignment) to $2.7 \mathrm{~mm}$ (with alignment by the ends). This implies that the interconnections and the correctors will be mostly within tolerance, 
TABLE $\mathrm{V}$

Average AND Standard DEVIATION IN RADial DisPlacemENTS FOR SeXtupole Position With New Alignment

\begin{tabular}{lccc}
\hline \hline & & \multicolumn{2}{c}{$\Delta \mathrm{R}[\mathrm{mm}]$} \\
& Dipoles & Average & St. dev. \\
\hline After manufacturing & 69 & +0.39 & 0.30 \\
Arrival at CERN & 42 & +0.31 & 0.20 \\
After cryostating & 30 & +0.30 & 0.21 \\
After cold test & 23 & +0.33 & 0.22 \\
\hline \hline
\end{tabular}

while the dipole mechanical axes will be at a radial offset up to $2.7 \mathrm{~mm}$.

\section{CONCLUSIONS}

We discussed here the geometrical properties of the dipoles recently built. We observed that in many cases these dipoles were too curved and some of them were out of tolerance so that a reshaping was tried to recover the nominal curvature. The re-shaping procedure, however, proved to be unstable and therefore has been omitted from the construction procedure. In order to recover dipoles with the extremities out of tolerance, we are investigating a possible strategy consisting in assembling them with the spool pieces mounted along the theoretical axis and aligning them by the ends. The resulting geometrical properties are then well within tolerance only the dipole center is shifted out of tolerance. More studies are needed to evaluate the consequences of this effect.

\section{ACKNOWLEDGMENT}

The authors would like to thank Marta Bajko, Dominique Missiaen, Juan Garcia Perez, Rocio Chamizo, Marco Buzio and Stephane Fartoukh, all working at CERN, for discussions and very useful suggestions.

\section{REFERENCES}

[1] R. Bartolini and W. Scandale, "Multipole feed-down due to dipole misalignments,", CERN LHC MMS Internal Note 97-12, 1997.

[2] M. D. Anerella et al., "Industrial production of RHIC magnets," IEEE Trans. Magn., vol. 32, no. 4, pp. 2059-2064, 1995.

[3] W. Scandale, M. Bajko, and F. Savary, "Geometry and alignment requirements for the LHC main dipole," in Proceeding EPAC2000, 2000, pp. $2160-2162$

[4] J. Garcia-Perez et al., "A novel device for the measurement of the mechanical and magnetic axes of superconducting magnet assemblies for accelerators," IEEE Trans. Appl. Supercond., vol. 12, no. 1, pp. 1731-1735, March 2002 